\title{
Improving venous thromboembolism risk assessment rates in a tertiary Ear, Nose and Throat Department
}

\author{
Authors: Manjit Dhamret and Rebecca McKnight
}

\section{Introduction}

Venous thromboembolism (VTE) is a significant cause of mortality and morbidity among hospitalised patients. A VTE risk assessment reduces this through facilitating correct prophylaxis. VTE accounts for approximately 25,000 in-hospital deaths in England annually. ${ }^{1}$ The cost to the NHS is estimated at $£ 640$ million/annum. ${ }^{2}$ Many of the risk factors are well known, such as advanced age, immobility, surgery, and obesity. The most important element of the VTE risk assessment strategy in England is to risk assess all patients for VTE on admission.

The aim of our quality improvement programme (QIP) was to explore the current practice of venous thromboembolism (VTE) prophylaxis within the Ear, Nose and Throat (ENT) Department. In addition to the accuracy and completion rates, we wanted to reduce the risk of VTE for ENT inpatients, and ultimately to educate healthcare professionals regarding VTE.

\section{Materials and methods}

Our standards were based on the 2018 National Institute for Health and Care Excellence and University Hospitals of North Midlands (UHNM) guidelines which state that all admitted patients must have a completed VTE assessment form within 24 hours of admission and receive appropriate VTE prophylaxis. The audit and re-audit were conducted over a period of 5 months. We utilised a pro forma to randomly assess the VTE forms of adult ENT inpatients. We individually analysed each section for completion, and also looked at whether VTE was prescribed correctly in lieu of patient presentation.

Our intervention included various teaching presentations individually targeted towards nurses, pharmacists and doctors. We presented the audit and re-audit findings at the ENT departmental meeting. We have since also presented the findings within the county acute medical unit (AMU).

\section{Results and discussion}

Phase 1 results demonstrated that we were generally good at signing each section (95.5\%) but not at completing the VTE in full (38.6\%). One particular area of concern was the completion of the VTE re-assessment at 24 hours which was $13.6 \%$. Phase 2 results demonstrated $100 \%$ completion in four areas, and $>90 \%$ completion in 9 out of 11 areas. Completion of the VTE in full improved to $72.2 \%$ and the re-assessment rates improved to $61.1 \%$.

\section{Conclusion}

We analysed the potential barriers to VTE completion and discovered three themes: initiative barriers, individual barriers and organisational barriers. More works needs to be done to educate staff members, especially those new to a trust. We also discussed measures such as checking the VTE on ward round and highlighted the need for re-assessment post-operatively. We have highlighted the difficulties in driving change in an established routine and will continue to develop teaching sessions to spread the word. We have been invited to speak at the UHNM VTE steering group.

\section{References}

1 Carrier M, Le Gal G, Wells PS, Rodger MA. Systematic review: casefatality rates of recurrent venous thromboembolism and major bleeding events among patients treated for venous thromboembolism. Ann Intern Med 2010;152:578-89.

2 Heit JA, Silverstein MD, Mohr DN et al. Predictors of survival after deep vein thrombosis and pulmonary embolism: a populationbased, cohort study. Arch Intern Med 1999;159:445-53. 